\title{
An Imaging Displacement Sensor with Nanometer Accuracy
}

\author{
David Woody $^{* a}$ and David Redding ${ }^{\mathrm{b}}$ \\ a Owens Valley Radio Observatory, California Institute of Technology, 100 Leighton Lane, Big Pine, CA, USA 93513- \\ 0968; 'JPL, 4800 Oak Grove Drive, Pasadena, CA, USA 91109
}

\begin{abstract}
An imaging displacement sensor (IDS) has been developed that can measure the displacement with an accuracy of 30 $\mathrm{nm}$ in $0.03 \mathrm{~s}$ with the precision improving to $1 \mathrm{~nm}$ for averaging times of $100 \mathrm{~s}$. The IDS consists simply of a light emitting diode (LED) pinhole collimator and a charge-coupled device (CCD) camera chip. The position accuracy is better than $0.05 \%$ over the few mm CCD size with deviation from linearity $<140 \mathrm{~nm}$. All six degrees of freedom (DoF), three translations and three angles, can be measured with the same accuracy by combining multiple IDS with different collimator beam orientations and knowing the nominal separation between the collimators and CCDs.
\end{abstract}

Keywords: metrology, sensors, telescope surface control

\section{INTRODUCTION}

Large telescopes with segmented primaries use "edge sensor" to measure the displacement of each segment relative to its neighbors. These measurements are utilized by the control system to maintain the surface figure. Custom designed versions of capacitive or inductive displacement sensors in a balanced bridge configuration are widely used for segment edge sensors ${ }^{1}$. The challenge for these sensors is to provide the required high precision for the desired degrees of freedom while minimizing the effect of lateral shifts of the segments in the plane of the surface and to operate over the wide temperature range and variable environment experienced by telescopes.

The sensor described in this paper was developed for CCAT, a $25 \mathrm{~m}$ diameter submillimeter-wave telescope that will operate inside a dome located on Cerro Chajnantor in the Atacama Desert ${ }^{2,3}$. The telescope must have high aperture efficiency at a wavelength of $350 \mu \mathrm{m}$ with good performance out to $200 \mu \mathrm{m}$. The telescope has 162 keystone shaped segments arranged in six rings with each segment mounted on three computer controlled actuators. The segment design is described in Woody et $\mathrm{al}^{4}$. A closed loop control approach employing edge sensors which measures the relative displacements between neighboring reflector segments has been developed by Redding et $\mathrm{al}^{5,6}$.

The critical requirement is to achieve a net $1 / 2$ wave front error (HWFE) of less than $10 \mu \mathrm{m}$, including the primary, secondary and tertiary optics. The top down error budget allocates only $6 \mu \mathrm{m}$ for the primary surface measurement and control, not including segment fabrication and distortion errors. CCAT differs from a large optical telescope in a couple of important aspects. One difference is that only a few of the planets are bright enough and in the correct size range at submillimeter wavelengths to be used for measuring the wavefront and routine measurements of the wavefront to set the reference position for the edge sensors is not feasible for CCAT. Another difference is that although the segments will utilize carbon fiber reinforced plastic (CFRP) subframes to provide a very stiff and stable structure, their stiffness and CTE is more than an order of magnitude worse than the ULE material used for optical telescopes. A more mundane but important distinction is that the budget for radio and submillimeter telescopes is an order of magnitude less than for comparably sized optical telescope, hence the sensors must be inexpensive.

There are a variety of sensors that have been used or are proposed for use in applications like CCAT. Laser metrology systems tend to be overly complicated, expensive and don't tolerate interruption of the light beam or power. Lateral position measurements systems using lasers and quadrant detectors can operate over large distances but have difficulty achieving better that $1 \mu \mathrm{m}$ precision and long term stability because of the temperature effects on the many components

*dwoody@caltech.edu; phone 1 760-938-2075x111; fax 1 760-938-2075; http://www.ccatobservatory.org

Modern Technologies in Space- and Ground-based Telescopes and Instrumentation II, edited by Ramón Navarro, Colin R. Cunningham, Eric Prieto, Proc. of SPIE Vol. 8450, $84500 \mathrm{U}$ (C) 2012 SPIE · CCC code: 0277-786X/12/\$18 - doi: 10.1117/12.925231 
with different material properties. Interdigitated capacitive and inductive sensors can be configured to measure lateral displacements but it is difficult to separate out the dependence on gap spacing and dihedral angle from the desired lateral or piston measurements. Standard capacitive or inductive sensors used to measure the distances between parallel plates can be arranged on cantilevered "paddles" can measure the piston motion between segments with minimum sensitivity to the gap separation between segments. Pairs of such sensors with appropriate cantilever distances can measure both the dihedral angle and piston displacements as is done on the Keck telescopes ${ }^{7}$.

Simulation and analysis of the control system indicates that the CCAT primary surface can be maintained at the required accuracy under the expected gravitational and thermal load conditions if the sensors errors are less than $100 \mathrm{~nm}$, remain stable for many months and the six DoF displacements are measured between neighboring segments. ${ }^{5,6}$ Measuring all six DoF at the edge between segments implies many more individual sensors are needed and is a new requirement beyond what is used or proposed for optical telescopes. So despite the allowable surface error for CCAT being a factor of a hundred larger than for an optical telescope the sensors are still challenging and the previously used edge sensors and proposed new designs for optical telescopes do not meet all of the CCAT requirements.

The ideal sensor would be a simple inexpensive system that unambiguously measures the six DoF displacements between two segments. Such a sensor system could find wide applicability to other tasks such as measuring the distortions, creep or strain is structures and precise positioning of probes or components. This paper describes the design and performance of an IDS that meets these requirements. The basic IDS is described in the next section. The IDS uses a new imaging processing algorithm which is described in section 3. The performance and test results of a prototype IDS are presented in section 4 . The conclusions and summary are given in section 5.

\section{SENSOR DESIGN}

The IDS is based on the simple idea of using a CCD camera to measure the position of a collimated beam. Excellent precision is achieved by using a shadow mask or pinhole collimator and exploiting the stable structure and accurate lithography of CCD chips. A schematic cross-section of the IDS is shown in Figure 1. A prototype IDS was fabricated from aluminum to evaluate the performance and capabilities of the IDS concept.

The collimator consists of a small light source located at one end of the collimator tube a pinhole or slit aperture at the other end. Black paint and baffles minimize the stray light and reflections in the collimator and along the light path to the CCD. In addition the collimator housing, aperture and light source mounting plate are all fabricated from the same material to minimize the effect of temperature changes.

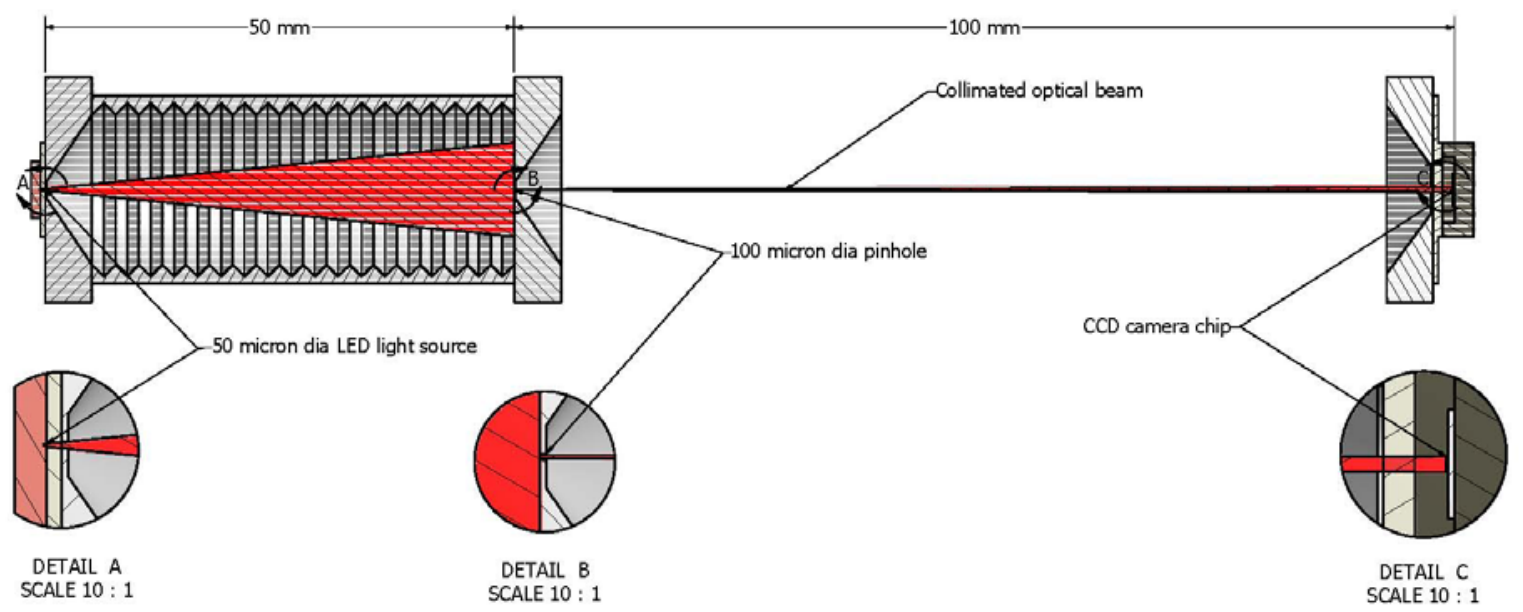

Figure 1. IDS schematic. The LED light source and collimator are on the left and the CCD is on the right.

The prototype IDS collimator uses a surface emitting LED for the light source to illuminate a $100 \mu \mathrm{m}$ diameter pin hole in a thin metallic sheet $100 \mathrm{~mm}$ from the LED. A multimode fiber optics communication LED is utilized which has a 50 $\mu \mathrm{m}$ diameter emitting area that is accurately defined by the photolithography of the electrode on the surface. The active 
LED is embedded in a clear plastic package with a dome lens. The dome lens is ground flat and polished to give a good optical interface and the LED is bonded to a microscope cover slip. This is then fastened to the collimator end plate which has a $100 \mu \mathrm{m}$ diameter aperture to limit scattered light from the LED. All of the metal parts and the non-emitting sides of the LED package are painted black to minimize internal reflections and stray light leakage. The prototype is fabricated as a box structure from $6.3 \mathrm{~mm}$ thick aluminum plates and lined with felt to minimize reflections. A laser diode which was much brighter than LED was tried but the temperature and bias dependence of the laser intensity and speckle pattern noise significantly degraded the IDS performance.

This collimator design is very simple and inexpensive and avoids the thermal and mechanical stability problems associated with mounting of glass optical components that are used in standard optical collimators. The small pinhole produces a beam divergence that is dominated by diffraction which has the advantage that the spot pattern is symmetric even if the aperture is not. As will be seen in the analysis presented in the next section this is critical for meeting the required performance goals.

The prototype IDS uses a commercial CCD single board camera [Sentech STC-TB33USB-B]. The CCD in this camera is a monochrome $640 \times 4807.2 \mu \mathrm{m}$ square pixel Sony chip. The pixels saturate at $\sim 50,000$ electrons and the standard 8bit output is a good match to the photon counting statistics. Measurements that do not require the nm level of precision demonstrated here could use cheaper CMOS based cameras. The images typically are captured at a rate of 30 frames/s in raw "tif" format over a USB cable. The collimated beam is bright enough to saturate the center of the spot image. The maximum precision is obtained by coadding multiple frames to decrease the effect of photon counting noise.

The CCD chip in the camera is attached directly to the IDS mounting plate to avoid distortions from the chip packaging and circuit board. The prototype IDS used a precision machined mounting plate designed to hold the perimeter of the CCD chip. The mount was gently stressed to allow insertion of the chip and removing the stress resulted in a clamping force around the perimeter of the chip. A simpler design is to bond the glass front of the CCD chip to a quartz microscope cover slip and then bond the cover slip to the mounting plate.

\subsection{Multiple IDS configurations}

A single IDS measures two degrees of freedom of the displacement of the CCD relative to the collimator. The spot motion could arise either from simple lateral $\mathrm{X}$ and $\mathrm{Y}$ shifts or from rotation about the $\mathrm{X}$ and $\mathrm{Y}$ axes at the collimator, where the collimator beam defines the Z-axis. The distinction between lateral shifts and rotations is critical in the edge sensor application of measuring and correcting the primary surface of a telescope. The use of IDS pairs as shown in Figure 2 removes the ambiguity and measures four degrees of freedom, lateral shifts in $\mathrm{X}$ and $\mathrm{Y}$ as well as dihedral angle rotations about the $\mathrm{X}$ and $\mathrm{Y}$ axes. Referring to a coordinate system centered half way between the collimator and CCD, small lateral shifts and dihedral angle rotations of $\mathrm{B}$ relative to $\mathrm{A}$ are given by

$$
\begin{gathered}
\Delta X=\frac{X_{A}+X_{B}}{2} \\
\Delta Y=\frac{Y_{A}-Y_{B}}{2} \\
\Delta \theta_{X}=\frac{Y_{A}+Y_{B}}{L_{1}} \\
\Delta \theta_{Y}=\frac{X_{A}-X_{B}}{L_{1}}
\end{gathered}
$$

where the $A$ and $B$ subscripts refer to the spot positions on the two CCDs and $L_{1}$ is the distance from the pinhole to the CCD. Note that $\mathrm{X}$ and $\mathrm{Y}$ direction for the image positions are in the coordinate system of the CCDs shown in green in Figure 2. 


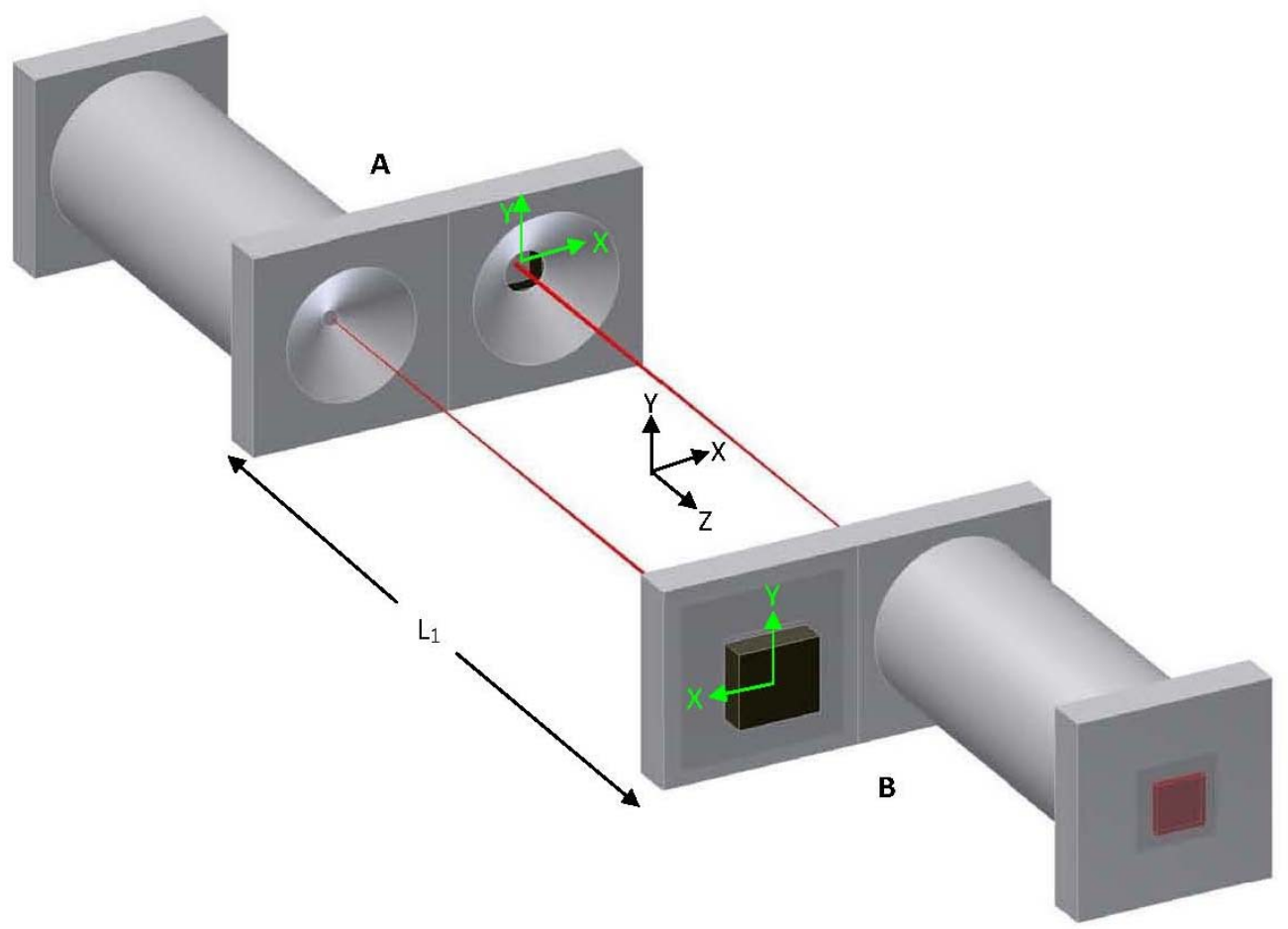

Figure 2. A pair of IDSs configured to measure four degrees of freedom, displacements in $\mathrm{X}$ and $\mathrm{Y}$ in the plane perpendicular to the collimated beam direction and the dihedral angle rotations about the $\mathrm{X}$ and $\mathrm{Y}$ axes shown in black. The CCD coordinate system for the image positions are shown in green

All six DoF can be measured using two IDS pairs configured as shown in the Figure 3. The two IDS pairs are oriented at an angle of $\pm \emptyset$ relative to the boundary between the two components whose relative displacements are being measured. Small 6 DOF displacements can be deduced from the readings from the two IDS pairs using

$$
\begin{gathered}
\Delta X_{\text {comp } 1}=\frac{\Delta X_{\text {left }}+\Delta X_{\text {right }}}{2 \sin \emptyset} \\
\Delta Y_{\text {comp } 1}=\frac{\Delta Y_{\text {left }}+\Delta Y_{\text {right }}}{2} \\
\Delta Z_{\text {comp } 1}=\frac{\Delta X_{\text {left }}-\Delta X_{\text {right }}}{2 \cos \emptyset} \\
\Delta \theta_{\text {Xcomp } 1}=\frac{\Delta \theta_{\text {Xleft }}+\Delta \theta_{\text {Xright }}}{2 \sin \emptyset} \\
\Delta \theta_{\text {Ycomp } 1}=\frac{\Delta \theta_{\text {Yleft }}+\Delta \theta_{\text {Yright }}}{2} \\
\Delta \theta_{\text {Zcomp } 1}=\frac{\Delta Y_{\text {left }}-\Delta Y_{\text {right }}}{L_{2}}
\end{gathered}
$$

where $L_{2}$ is the separation of the two IDS pairs along the boundary between component 1 and 2 and $L_{2} \gg L_{1}$. The left and right subscripts refer to the left and right IDS pairs in Figure 3. The measured quantities on the right side of the equations are in the reference frames shown in black in Figure 3 of the IDS left and right pairs respectively. A proper control system would encapsulate the exact geometry of the IDS configuration and utilize the calculated response of the four CCD image positions for the six DoF displacements. There are eight readings from the two IDS pairs and only six 
independent displacements leaving effectively two extra measurements that can be used to test for internal consistency or distortion of the components.

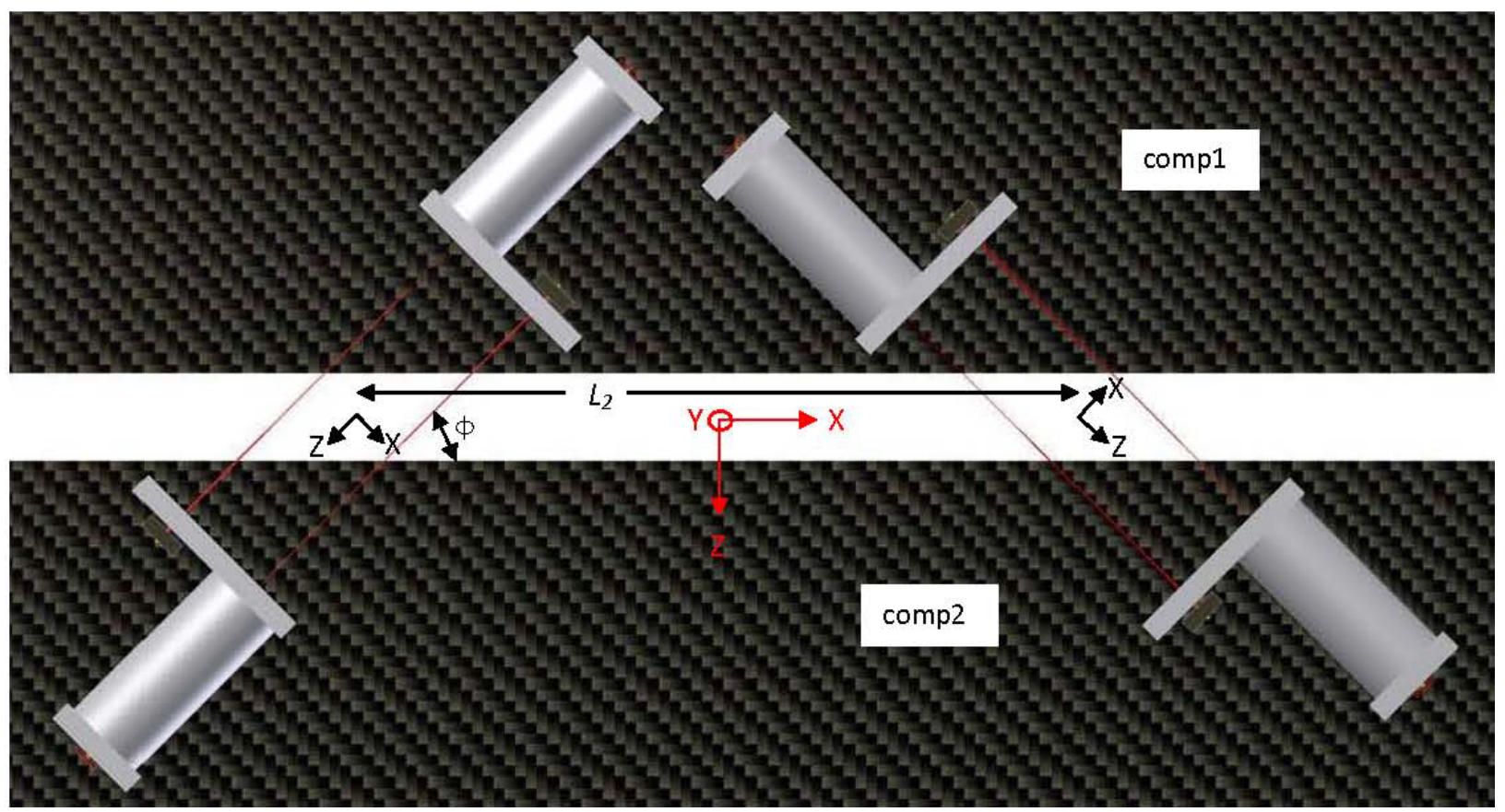

Figure 3. Two IDS pairs configured to measure 6 DoF displacements between two components.

In the case where measurement of lateral displacement in only one direction is required, the pinhole in the collimator can be replaced by a slit and the position of the line image can be determined in one direction on the CCD. A line image has many more pixels in the critical transition from dark to saturation which decreases the effect of photon counting noise in the direction perpendicular to line. An IDS with a slit collimator can also serve as a high resolution non-contacting angle encoder by measuring the angular orientation of the line image on the CCD.

\section{CENTROID ALGORITHM AND SIMULATIONS}

There are a large variety of algorithms for solving the center position of a spot but many of these are aimed at finding the center of a dim spot with only a few photons detected or only need to produce answers accurate to a modest fraction of a pixel. The IDS CCD spot image ideally is saturated with high contrast and covers many pixels. The IDS image processing emphasis is on producing an accurate and robust determination of the centroid position with an uncertainty that is dominated by the photon counting statistics.

\subsection{Image preprocessing}

The first step in the processing is to co-add multiple image frames together to improve the photon statistics. For a typical application requiring $1 \mathrm{~s}$ time resolution, 30 video frames taken at 30 frames/s averaged together decreases the photon counting noise by more than a factor of five. Depending upon the CCD camera's capabilities the time resolution or counting statistics can be further improved by increasing the video frame rate along with a corresponding increase in the beam intensity to keep the spot image saturated in each frame.

An important aspect for the image processing is to minimize the effects of the background light, dark current, readout noise and the pixel responsivity. These effects can be measured by turning the collimated beam LED off and using a variable current LED mounted on the front of the pinhole aperture plate to illuminate the full CCD. The flat field response can reveal dirt on the CCD and used to correct the CCD pixel response and minimize its effect on determining the centroid position. 
An alternate approach which was used for the prototype IDS was to enhance the contrast in the co-added images to minimize the dark field and response effects. The normalized pixel readings were recomputed using

$$
\text { Pix } x_{\text {after }}=\frac{1}{1+\mathrm{e}^{- \text {Gain }\left(2 \text { Pix }_{\text {before }}{ }^{-1)}\right.}}
$$

where Pix $x_{\text {before }}$ is the normalized pixel reading in the image with a range from 0 to 1 . This yields an "S" shaped response which amplifies the differential response for the mid-range transition pixels by the Gain factor and suppresses the response at the dark and saturated ends of the range. The images from the prototype sensor have been processed using Gain=5. Figure 4 shows a typical image from the prototype IDS before and after this contrast enhancement. The low level effects such as the dark current variations and diffraction pattern ripples are greatly reduced in the contrast enhanced image.
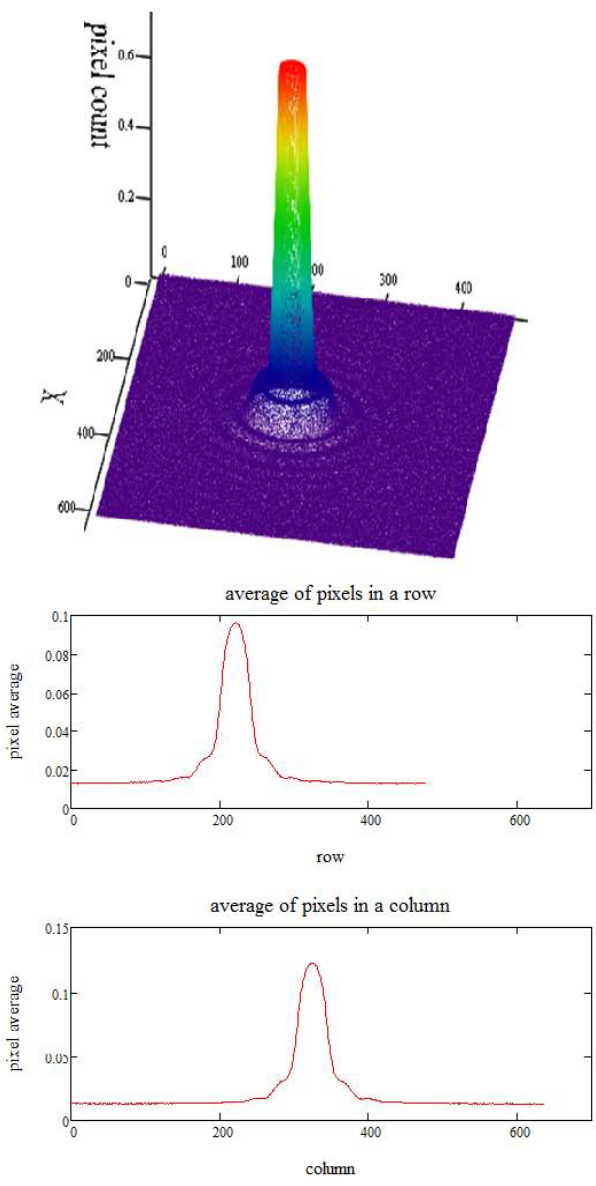
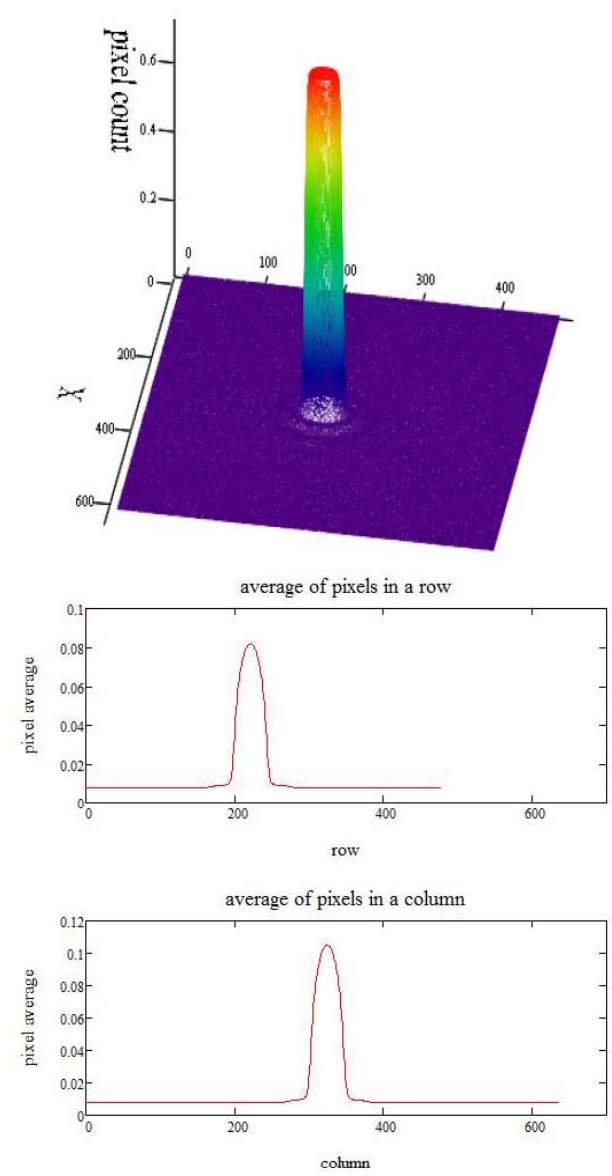

Figure 4. Typical IDS CCD image before (left) and after contrast enhancement with a Gain of 5 (right). The bottom plots show the row and column pixel averages.

\subsection{Centroid position}

The easiest method for estimating the position of the image spot is to simply calculate the center of mass of the image. This is quick and efficient but the non-uniform background and weak tails of the spot image make the results dependent on the beam brightness and size. Adding a lower bound threshold can reduce this effect but the calculated position still has some dependence on the chosen threshold level.

Another complication is that the apparent position of an asymmetric saturated image will change as the image brightness changes. Hence it is important to have the collimator beam produce a symmetric spot image. Using a high quality aperture will improve the symmetry but also using an incoherent light source and small pinhole so that the beam divergence is dominated by diffraction naturally produces a symmetric image independent of the aperture shape. 
The approach developed here is not to solve for the center of mass but to determine the symmetry point for the image. The symmetry point and the center of mass will be the same for a well behaved symmetric image and uniform background. Although the image is 2D it is sufficient to add the pixels in the rows and columns to produce two 1D curves of intensity vs. $\mathrm{X}$ and $\mathrm{Y}$ position as shown in Figure 4. One method for finding the symmetry point for a curve is to find the fold line that minimizes the deviation of one half of the curve compared to the folded version from the other half. This would work well if we were looking for modest sub-pixel resolution, but we are interested in solving for the symmetry point with a resolution of $<<1 / 100^{\text {th }}$ of a pixel which would require a very accurate interpolation between pixels and a fine grained search for the symmetry point.

The properties of Fourier Transforms (FT) offer a robust method for solving for the center of a symmetric curve. The phase of a single component of the complex FT of a 1D real data set determines the position of the peak of that sinusoidal component. Only the first component, which has a single period across the image, has an unambiguous peak position. The $\mathrm{N}^{\text {th }}$ component has $\mathrm{N}$ periods across the image and hence $\mathrm{N}$ possible positions for the peak. By starting with the first component and working sequentially to higher components and picking the nearest peak position that is closest to the previous peak, the true peak can be unambiguously tracked to the highest component which has one period across two pixels. The phase of any component is a continuous function of the position, i.e. not quantized, and the accuracy is only limited by the signal-to-noise $(\mathrm{S} / \mathrm{N})$ of that component even though the data are from discrete finite sized pixels. In the algorithm employed here the successive peak positions are added to a running sum weighted by the amplitude squared of that component. This accentuates the components with high signal-to-noise and down weights the weak components. Figure 5 shows the amplitude and positions (phase) for all of the components for the image shown in Figure 4 along with the weighted sum position. All of the components are in excellent agreement indicating that the image has excellent symmetry. An added benefit of this technique is that the standard deviation of the component positions is a measure of the image symmetry and can be used as a proxy for the expected accuracy of the spot centroid determination.

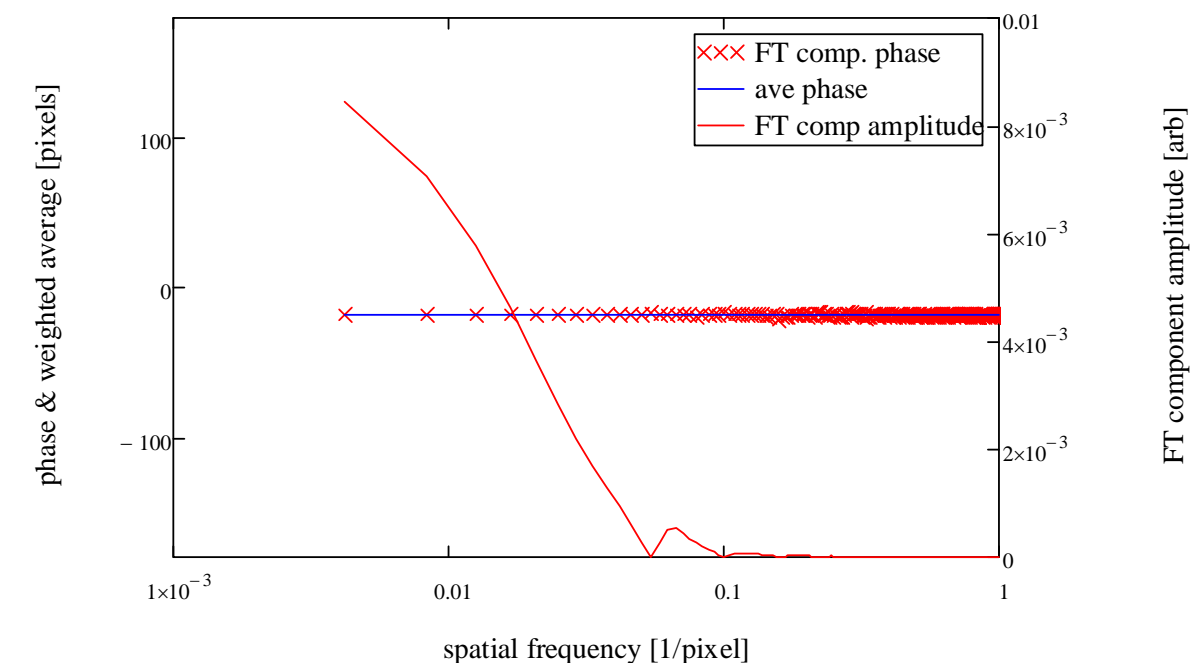

Figure 5. Fourier analysis of column averaged pixels from image show in Figure 4. The phase of each component has been converted to the equivalent position measured in pixels.

Simulations that included the photon statistics and dark current and 8-bit readout were used to calculate the expected performance. Simulations were also used to evaluate various centroid finding algorithms. The conclusions from the simulations are that the FT algorithm is expected to determine the centroid location with an accuracy of $\sim 1 / 100^{\text {th }}$ of a pixel for a saturated spot size diameter greater than 10 pixels and the center of a line image with even higher precision.

\section{PROTOYPE PERFORMANCE}

The prototype sensor was used to test the viability of the IDS concept and in particular to determine the achievable precision and long term stability. The LED, pinhole aperture and CCD were mounted on plates which could be placed at different positions to explore the performance and dependence on the spacing between the collimator and CCD. Initially a $100 \mu \mathrm{m}$ wide line aperture was used in the collimator to give better $\mathrm{S} / \mathrm{N}$ in one direction, but it was soon apparent that 
the $\mathrm{S} / \mathrm{N}$ and resulting precision were much better than required and a simple $100 \mu \mathrm{m}$ diameter pinhole was sufficient for our application. The results reported below use either a $100 \mathrm{~mm}$ or $200 \mathrm{~mm}$ spacing from the LED source to the pinhole aperture and $10 \mathrm{~mm}$ or $100 \mathrm{~mm}$ spacing from the pinhole to the CCD. The images were collected at a video rate of 30 frames/s and usually 150 frames are co-added to give $5 \mathrm{~s}$ averages.

\subsection{Dependence on LED brightness}

The initial test placed the CCD $10 \mathrm{~mm}$ from the collimator pinhole to minimize the effect of distortions of the aluminum housing while investigating the dependence on the collimator beam brightness. Figure 6 shows sample images for three different LED currents. Figure 7 shows the apparent spot position using the simple center-of-mass centroid method as a function of LED current. The plot shows systematic effects below $\sim 20 \mathrm{~mA}$ and a scatter of $\sim 0.2 \mu \mathrm{m}$ peak-to-peak for the $\mathrm{X}$ position and $\sim 0.08 \mu \mathrm{m}$ for the $\mathrm{Y}$ position. The scatter is much larger than can be explained by the photon counting statistics and are likely caused by low level asymmetric smear and ghost effects in the CCD and its readout. This would also explain the difference in scatter in the two directions since the transfer of charges and readout rates are different for the two axes. The center-of-mass centroid determination is very susceptible to these low level artifacts because they extend asymmetrically over many pixels.
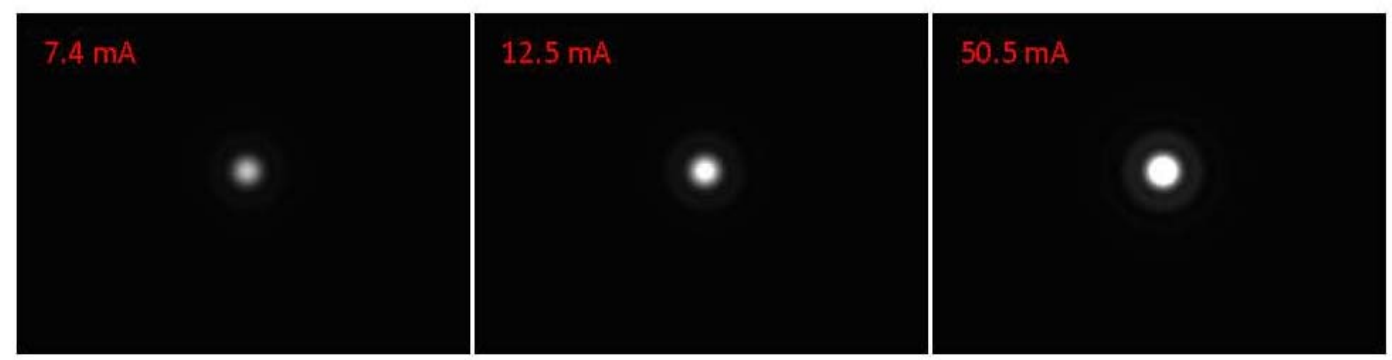

Figure 6. IDS images with collimator LED currents of 7.4, 12.5 and $50.5 \mathrm{~mA}$.

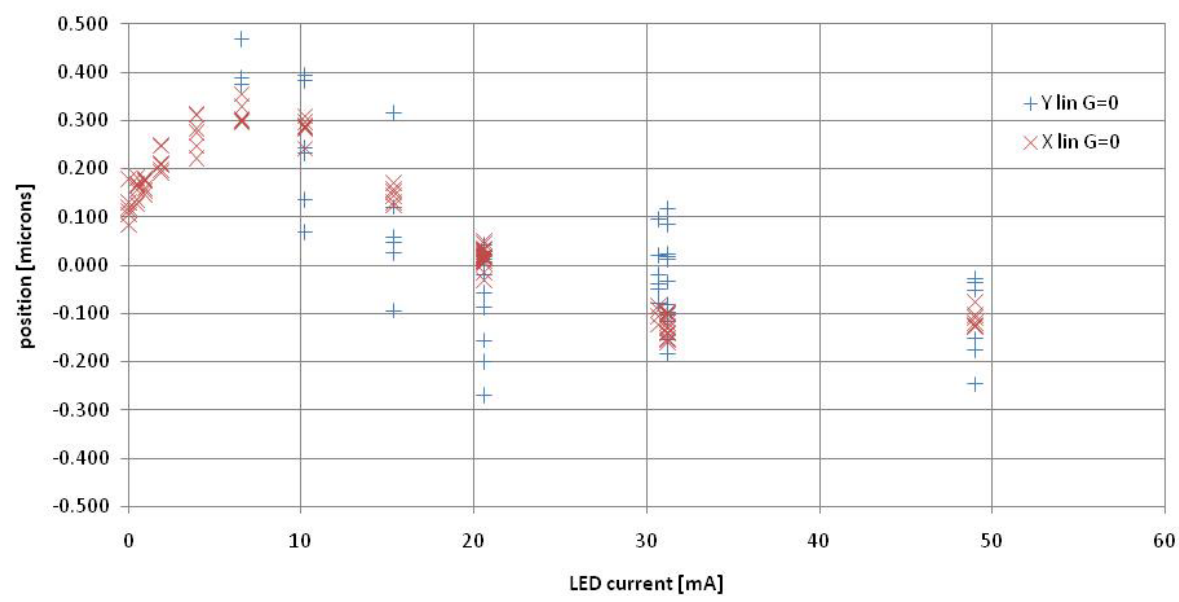

Figure 7. Deviation of the center-of-mass spot position from the ensemble average position vs. LED current. The data points are $5 \mathrm{~s}$ averages. The red X's are the $\mathrm{X}$ positions and the blue +'s are the Y positions.

Figure 8 shows the results for the same images processed using the FT symmetry algorithm described in section 3.2. The FT algorithm produced consistent results down to currents at low as $\sim 10 \mathrm{~mA}$ and the scatter is $\sim 10 \mathrm{~nm}$ peak-to-peak in both axes. The FT symmetry algorithm is very robust and has a precision that is consistent with the expected photon counting statistics with a very weak dependence on the collimator brightness.

\subsection{Temperature dependence}

The temperature stability will be critical for the application of the IDSs on CCAT. The operating temperature range is -20 to $+15 \mathrm{C}$ and the survival range is -30 to $+40 \mathrm{C}$. Figure 9 shows the IDS position reading as a function of temperature. The temperature dependence is $\sim 13 \mathrm{~nm} / \mathrm{C}$. The prototype IDS was assembled from bolted aluminum 
plates and it is expected that an IDS with a more monolithic design like that shown in Figure 1 and utilizing Invar or similar low thermal expansion material would have even better performance.

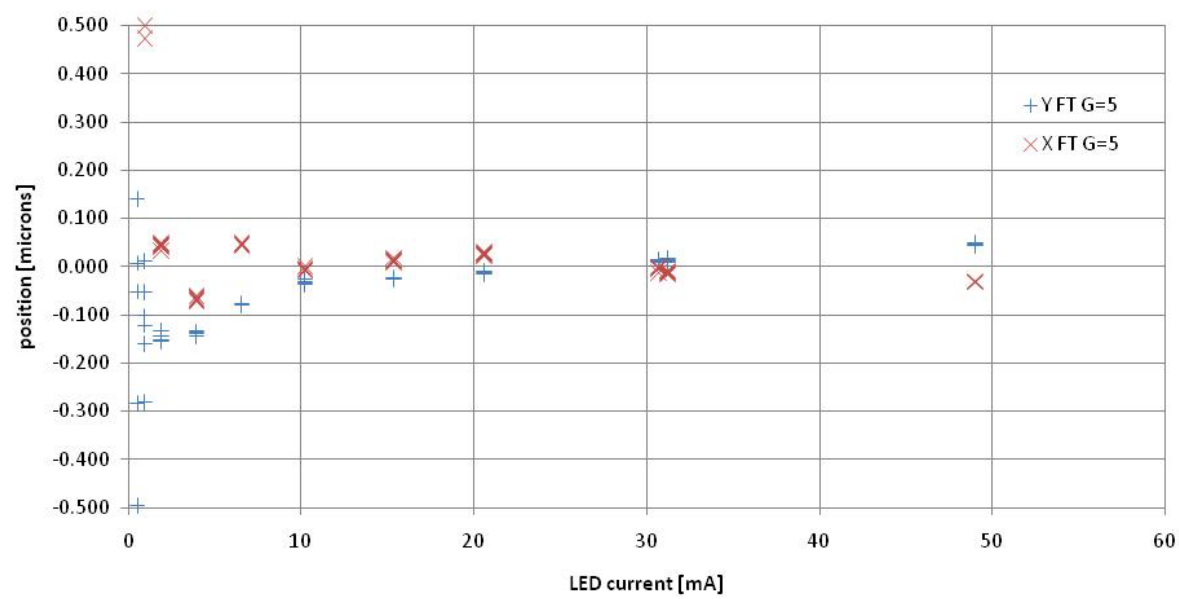

Figure 8. Deviation of the center-of-mass spot position from the ensemble average position vs. LED current for the same image data as Figure 7 but processed using the FT symmetry algorithm with a Gain of 5 in the contrast enhancement. The data points are $5 \mathrm{~s}$ averages. The red x's are the X positions and the blue +'s are the Y positions. The data at a single current setting are so close together that they look like a single point in the plot.

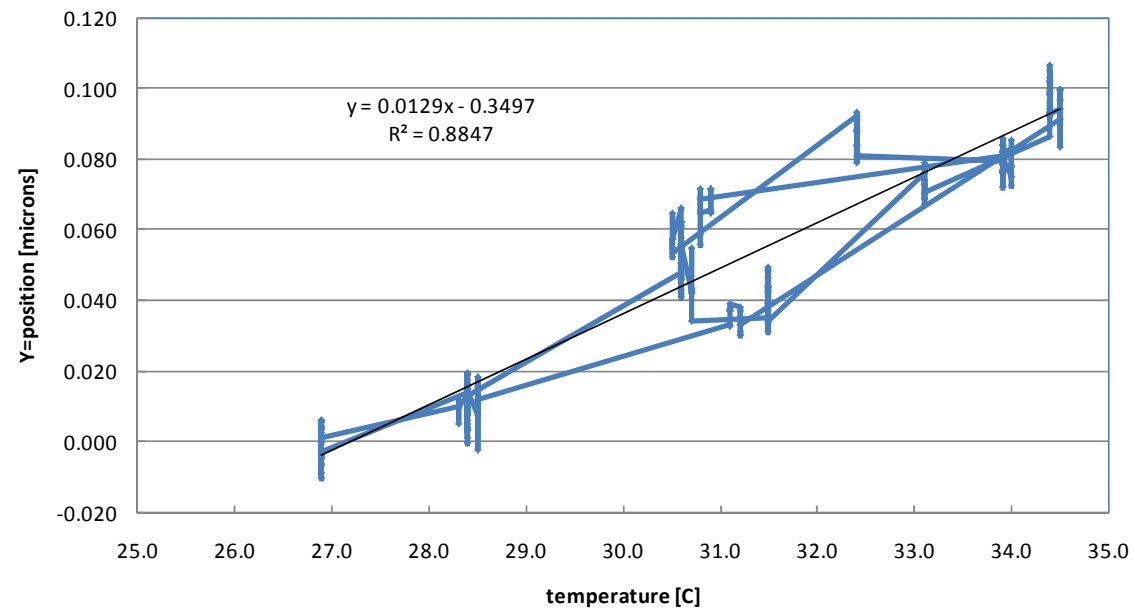

Figure 9. Plot of the IDS spot position as a function of temperature.

\subsection{Long term stability}

Calibrating or resetting a sensor zero position interrupts the system and can be impractical on a large telescope like CCAT. Thus long term stability is another important performance parameter. The IDS prototype with a pinhole to CCD spacing of $100 \mathrm{~mm}$ was monitored for four months to evaluate the long term stability. It was insulated from the lab environment and experienced $\sim 3$ C peak-to-peak temperature variations. The Allan deviation for the X and Y spot positions are plotted in Figure 10. The minimum Allan deviation is less than $1 \mathrm{~nm}$ for averaging times of 100-1000 sec. The deviation is larger for the Y position on short time scales, probably associated with the CCD readout effects, while on the longest time scales the $\mathrm{X}$ positions show larger deviations than the $\mathrm{Y}$ positions, most likely associated with temperature effects and the aluminum IDS housing construction. The systematic drift or aging effect is less than 50 $\mathrm{nm} / \mathrm{month}$. 


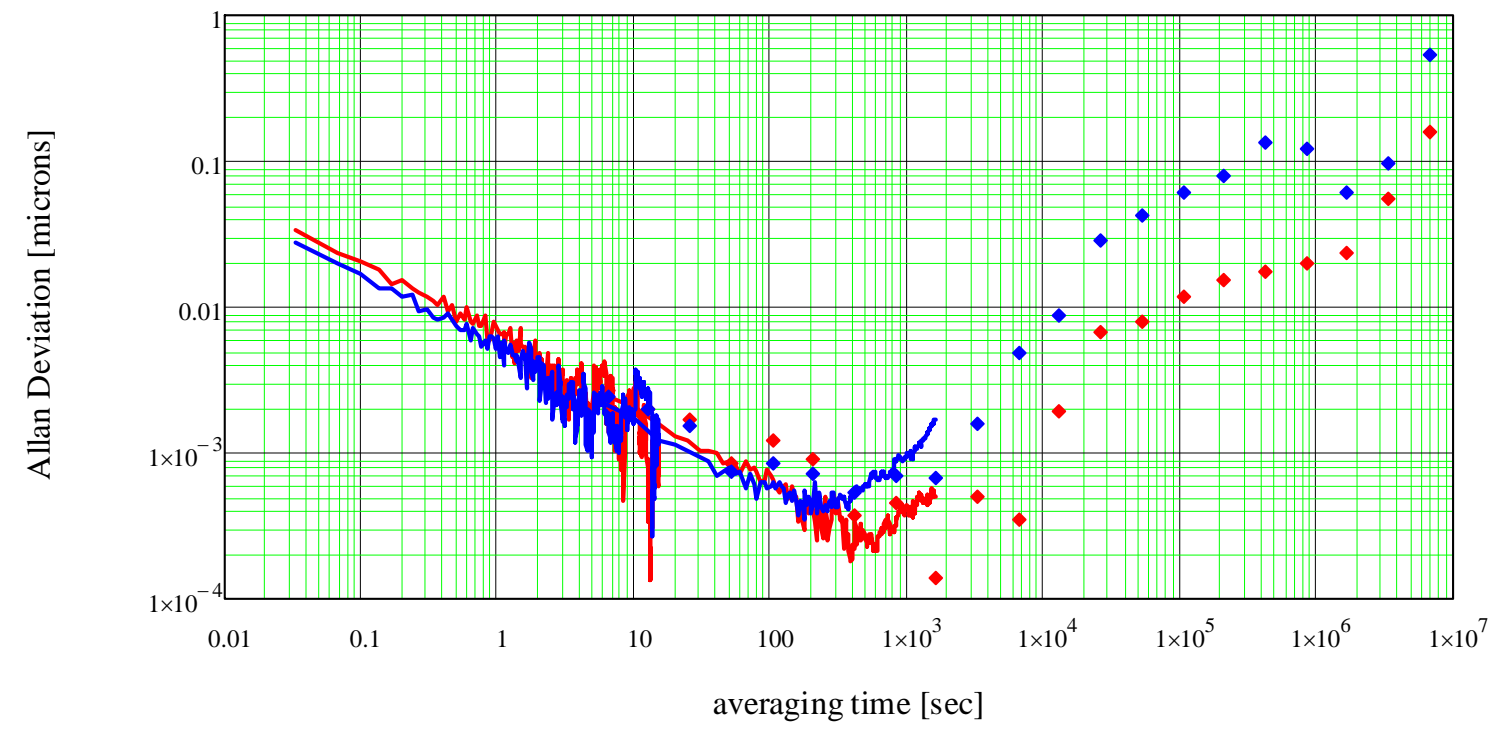

Figure 10. Allan deviation of X (blue) and Y (red) spot positions obtained over a four month period.

\subsection{Linearity and accuracy}

Measuring the linearity accuracy at the sub-micron level over a range of several millimeters usually requires an expensive metrology system in a temperature controlled environment. The method used here was to turn the IDS into a simple stationary pinhole telescope to observe the sun as the earth rotates and moves the image across the CCD at a precise $15 \mathrm{arcsec} / \mathrm{sec}$. All that is required is to remove the LED and its mounting plate from the IDS housing and point the housing at the sun so that the beam defined by the sun and pinhole lands on the CCD. A layer of aluminized Mylar was used to attenuate the sun at the pinhole and even then very short exposures of $1 \mathrm{~ms}$ were required to avoid complete saturation across the whole CCD. By holding the IDS fixed and knowing the pinhole to CCD spacing you will get a predictable motion of the sun's image across the CCD. The data consist of 5 minutes of video taken at 30 frames/s which is then processed to determine the average velocity along the sun track and any deviations from the expected track. These observations require a very clear day and good atmospheric seeing to minimize the image jitter, conditions which fortunately are common at the Owens Valley Radio Observatory where this work was performed.

Figure 11 shows the $\mathrm{X}$ and $\mathrm{Y}$ spot image positions as a function of the video frame number. The sun track was very linear with an rms deviation from a straight line of 0.36 and $0.27 \mu \mathrm{m}$ for the $\mathrm{X}$ and $\mathrm{Y}$ directions. The pinhole to CCD spacing was $90.4 \mathrm{~mm}$ giving a plate scale of $2.28 \mathrm{arcsec} / \mu \mathrm{m}$. Although the orientation of the telescope was not accurately known, the slopes for the $\mathrm{X}$ and $\mathrm{Y}$ motion combined yielded the expected rate of $15.000+-0.005$ arcsec/s with the uncertainty limited by the knowledge of the pinhole to CCD spacing. This measurement was repeated at different times during the day with different paths on the CCD. These measurements indicates that the absolute position and linearity is accurate to better than $0.05 \%$.

The track of the sun across the CCD remained well behaved and linear out to the edge of the chip where the large 0.9 $\mathrm{mm}$ diameter image of the sun started to touch the edge of the CCD. The $4.6 \mathrm{~mm} \times 3.5 \mathrm{~mm}$ CCD provides a $3 \mathrm{~mm} \times 4$ mm working range with full accuracy.

The pinhole to CCD spacing was reduced to $20 \mathrm{~mm}$ to produce a slower sun track across the CCD to look for small pixel size irregularities or periodicities. Figure 12 shows the deviations from a straight line for the track. The $20 \mathrm{~mm}$ spacing gives a plate scale of $10 \mathrm{arcsec} / \mu \mathrm{m}$ so the atmospheric seeing jitter is less than in Figure 11 but still dominates the deviation from a perfectly linear track. A Fourier analysis of the data in Figure 12 places an upper limit on any periodic structure in the errors of less than $30 \mathrm{~nm}$. 

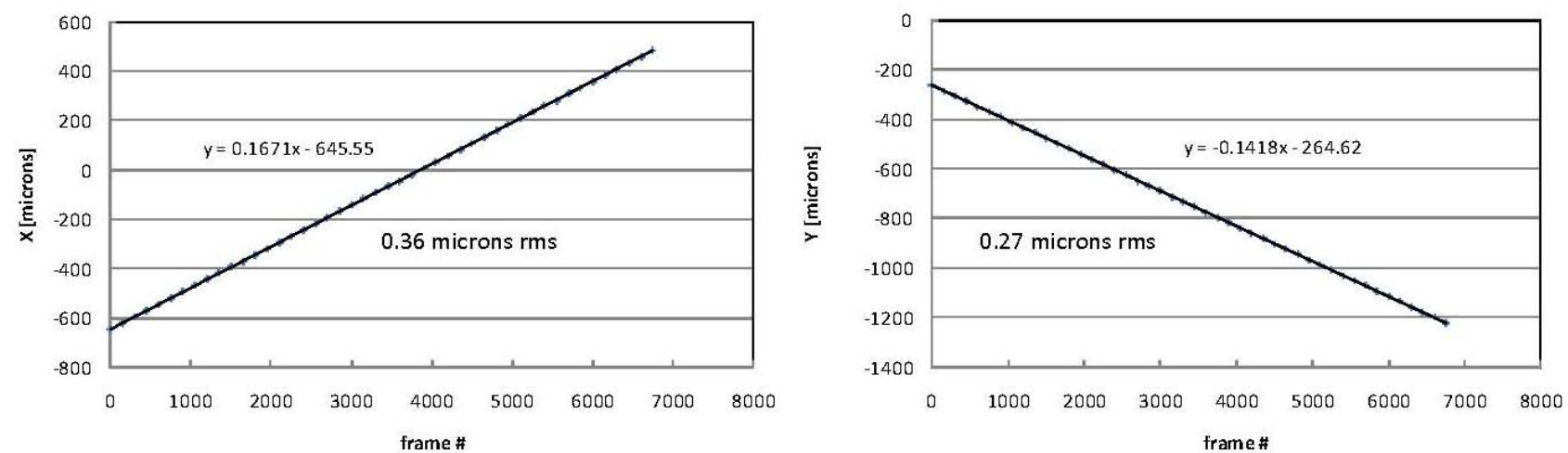

Figure 11. Sun track across CCD for pinhole to CCD spacing of $90.4 \mathrm{~mm}$. The plate scale is 2.28 arcsec/ $\mu \mathrm{m}$ and the atmospheric seeing dominates the noise in the linear fit.
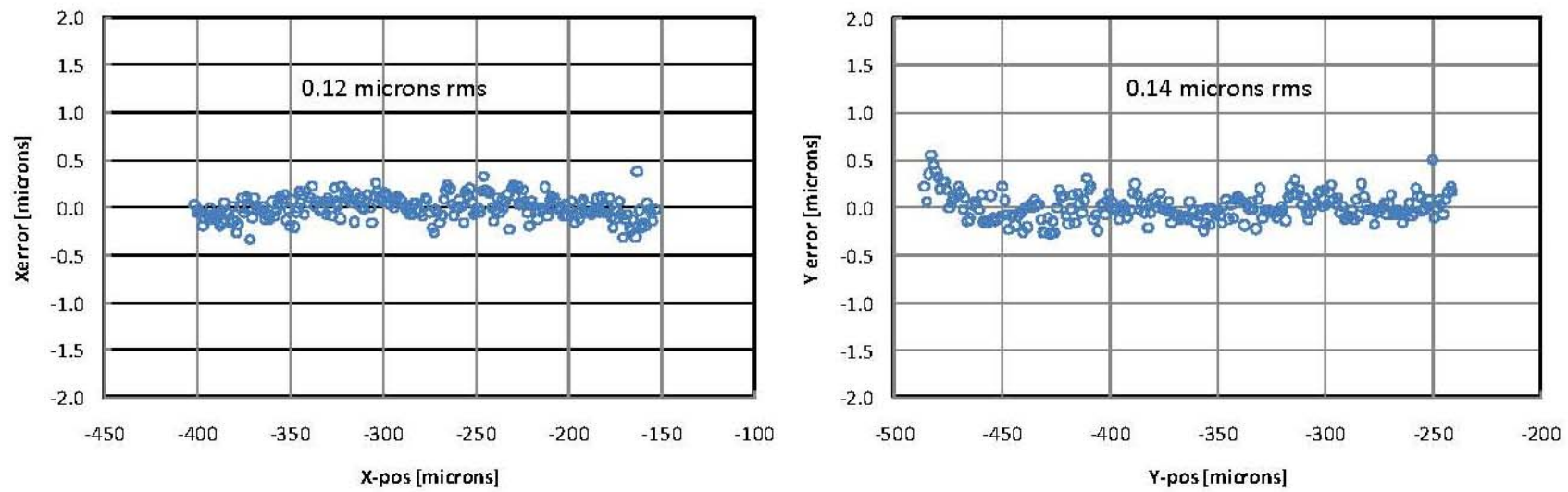

Figure 12. Sun track across CCD for pinhole to CCD spacing of $20 \mathrm{~mm}$. The plate scale is 10 arcsec/ $\mu \mathrm{m}$ and the atmospheric seeing dominates the noise in the linear fit.

\section{SUMMARY}

A novel displacement sensor that can achieve nm precision and better than $0.05 \%$ accuracy over a $4 \mathrm{~mm}$ x $3 \mathrm{~mm}$ working area has been developed and evaluated. The IDS utilizes a very simple LED and pinhole collimator plus a commercial CCD board camera to measure the lateral displacement of the collimated beam. The sensor exploits the nm accuracy achieved in modern semiconductor fabrication. The components are inexpensive and easy to assemble into a robust high precision measuring system.

A FT based algorithm that precisely determines the symmetry point of the image was developed for measuring the position of the beam spot on the CCD. Simulations and tests of a prototype IDS demonstrate that the algorithm is insensitive to variations in the collimated beam brightness. The spot location for a single image is routinely better than $1 / 100^{\text {th }}$ of a pixel consistent with the photon counting statistics. The resolution improves to $\sim 1 / 10,000^{\text {th }}$ of a pixel when 10,000 images are combined.

The evaluation of the prototype IDS indicates that the temperature and long term stability are excellent. The temperature measurements were limited by the construction and CTE of the aluminum housing but still yielded a temperature dependence of only $13 \mathrm{~nm} / \mathrm{C}$. A more monolithic construction from a low expansion material like Invar is expected to decrease the temperature dependence by a factor of ten. The Allan deviation obtained from measurements over a four month period decreased from $30 \mathrm{~nm}$ on the $1 / 30^{\text {th }} \mathrm{s}$ time scale to a minimum of $0.5 \mathrm{~nm}$ on the 5 minute time scale and increasing to $100 \mathrm{~nm}$ over four months. 
The IDS accuracy was evaluated by removing the LED light source and using the IDS as a pinhole telescope. The IDS was held fixed while the pinhole image of the sun swept across the CCD. The earth rotation rate and the distance from the pinhole to the CCD give a very precise image speed across the CCD. This simple method suffers from atmospheric seeing effects but placed useful upper limits on the linearity and absolute accuracy of better than $0.05 \%$.

The IDS is a very flexible measurement tool. It can be tailored to applications requiring different measurement ranges and resolutions by using CCDs with different pixel sizes and number of pixels. The effective readout rate can be increased by using brighter LEDs and faster video rates. The angular sensitivity and range can be adjusted by changing the collimator to CCD spacing. The pinhole aperture size, LED wavelength and brightness can also be optimized for operation at working distances ranging from a few $\mathrm{mm}$ to $\sim 1 \mathrm{~m}$. Multiple IDSs can be combined to measure several degrees of freedom and a measurement system that measures the 6 DoF displacements between two components can be assembled from 4 IDSs. Potential applications for an IDS range from laboratory component displacement measurements to primary mirror segment to segment measurements and control on large telescopes with hundreds of segments.

\section{ACKNOWLEDGEMENTS}

This work was supported by the John B. and Nelly Kilroy Foundation.

\section{REFERENCES}

[1] Mast, T.S., et al., An Edge Sensor Design for the TMT. Proc. SPIE. 6267 (2006).

[2] Sebring, T.A., The Cornell Caltech Atacama Telescope: progress and plans 2010. Proc. SPIE. 7733: p. 77331X-177331X-11 (2010).

[3] Woody, D., et al., The CCAT 25 m diameter submillimeter-wave telescope. Proc. SPIE. 8444 (2012).

[4] Woody, D.P., et al., Panel options for large precision radio telescopes. Proc. SPIE. 7018: p. 70180T1-70180T11 (2008).

[5] Redding, D.C., et al., Wavefront controls for a large submillimeter-wave observatory Proc. SPIE. 7733: p. 7733291-773329-11 (2010).

[6] Lou, J.Z., et al., Modeling a large submillimeter-wave observatory Proc. SPIE. 7733: p. 773326-1-773326-13 (2010).

[7] Mast, T.S. and J.E. Nelson, The Figure Control of Segmented Telescope Mirrors, in Keck Observatory Reports. 1983, Keck Observatory. 